\title{
GENERIC PROPERTIES OF THE COMPLEMENTARITY PROBLEM *
}

\author{
Romesh SAIGAL \\ University of California, Berkeley, Calif., U.S.A. \\ and \\ Carl SIMON \\ University of California, Berkeley, Calif., U.S.A. \\ University of Michigan, Ann Arbor, Mich., U.S.A. \\ Received 4 April 1972 \\ Revised manuscript received 9 February 1973
}

Given $f: \mathbf{R}_{+}^{n} \rightarrow \mathbf{R}^{n}$, the complementarity problem is to find a solution to $x \geq 0, f(x) \geq 0$, and $\langle x, f(x)\rangle=0$. Under the condition that $f$ is continuously differentiable, we prove that for a generic set of such an $f$, the problem has a discrete solution set. Also, under a set of generic nondegeneracy conditions and a condition that implies existence, we prove that the problem has an odd number of solutions.

Let $\mathbf{R}^{n}$ denote $n$-dimensional Euclidean space with the inner product

$$
\langle x, y\rangle=\sum_{i=1}^{n} x_{i} y_{i}
$$

Let

$$
\mathbf{R}_{+}^{n}=\left\{x=\left(x_{1}, \ldots, x_{n}\right): x \geq 0\right\},
$$

where $x \geq 0$ stands for $x_{i} \geq 0$ for all $i=1, \ldots, n$. Given a map $f: \mathbf{R}_{+}^{n} \rightarrow \mathbf{R}^{n}$, the complementarity problem is to find a solution to the system

\footnotetext{
* This work was partially supported by N.S.F. Grants GP-8007 and 010185.
} 


$$
x \geq 0, \quad f(x) \geq 0, \quad\langle x, f(x)\rangle=0 .
$$

It is a unified form of problems arising in such fields as mathematical programming $[3,9,14]$, game theory $[3,12,14]$, economic equilibrium theory [4, 12], mechanics [11], and geometry [8].

The special case where $f$ is affine, i.e., $f(x)=M x+q$, where $M$ is an $n \times n$ matrix and $q \in \mathrm{R}^{n}$, is called the linear complementarity problem and has been extensively studied in the literature. Among the more important results are those showing that in certain cases the special constructive procedures developed by Cottle and Dantzig [3] and Lemke [14] will actually compute a solution of the problem (1). Using these procedures, one can establish some results on the parity of the number of solutions of (1), as done by Lemke [14]. Some generic properties of the linear problem are known; for example, Murty [15] has shown that the linear problem has a finite (and thus discrete) solution set for all $q$ if and only if all the principal minors of $M$ are non-zero - clearly a generic property of matrices.

Most results of the non-linear complementarity problem are related to establishing the existence of a solution, for example, Cottle [2] and Karamardian [13]. In Section 3 of this paper, we will show that if $f$ is continuously differentiable $\left(\mathrm{C}^{1}\right)$, satisfies a couple of generic non-degeneracy conditions and an additional condition guaranteeing the existence of a solution, then (1) has an odd number of solutions. In Section 2 , using transversality arguments, we will show that for almost all $\mathrm{C}^{1}$ maps $f$, (1) has a discrete solution set.

Our methods were motivated by the similar results of Debreu [4] and Dierker $[5,6]$ in economics and by the philosophy of Smale [18] in applying global analysis. In addition, we would like to acknowledge helpful conversations with J. Heitsch.

In this section, we shall show that for almost all continuously differentiable maps $f: \mathbf{R}_{+}^{n} \rightarrow \mathbf{R}^{n}$, the solution set of the complementarity problem is discrete. To avoid any smoothness difficulties and to make our notation simpler, we shall work with $\mathrm{C}^{1}$ maps $\mathbf{R}^{n} \rightarrow \mathbf{R}^{n}$. We can do this because any $\mathbf{C}^{1}$ map $\mathbf{R}_{+}^{n} \rightarrow \mathbf{R}^{n}$ can be extended as a $\mathbf{C}^{1}$ map on all of $\mathbf{R}^{n}$ as follows: Let $f_{0}\left(x_{1}, \ldots, x_{n}\right)=f$ on $\mathbf{R}_{+}^{n}$. Given $f_{i-1}$ defined on $\left\{\left(x_{1}, \ldots, x_{n}\right): x_{i} \geq 0, \ldots, x_{n} \geq 0\right\}$, define $f_{i}$ on $\left\{\left(x_{1}, \ldots, x_{n}\right): x_{i+1} \geq 0, \ldots\right.$, $\left.x_{n} \geq 0\right\}$ by 


$$
\begin{aligned}
f_{i}\left(x_{1}, \ldots, x_{n}\right)= & \begin{cases}f_{i-1}\left(x_{1}, \ldots, x_{n}\right) & \text { if } x_{i} \geq 0 \\
& -f_{i-1}\left(x_{1}, \ldots, x_{i-1},-x_{i}, x_{i+1}, \ldots, x_{n}\right)+\end{cases} \\
& +2 f_{i-1}\left(x_{1}, \ldots, x_{i-1}, 0, x_{i+1}, \ldots, x_{n}\right) \text { if } x_{i} \leq 0
\end{aligned}
$$

$f_{i+1}$ extends $f_{i} \mathrm{C}^{1}$ and so $f_{n}$ extends $f$ to all of $\mathbf{R}^{n}$. See also [1, Appendix A].

Let $\mathrm{C}^{1}\left(\mathbf{R}^{n}, \mathbf{R}^{n}\right)$ be the vector space of $\mathrm{C}^{1}$ functions $\mathbf{R}^{n} \rightarrow \mathbf{R}^{n}$, given the topology of uniform convergence of both $f$ and its derivative $\mathrm{D} f$ on compact sets, $\mathrm{D} f(x)$ being the $n \times n$ matrix $\partial f_{i}(x) / \partial x_{j} . \mathrm{C}^{1}\left(\mathbf{R}^{n}, \mathbf{R}^{n}\right)$ is then a complete metric space, and by the Baire Category Theorem any residual subset of it is dense. Recall that a subset $U$ of a topological space is residual if $U$ contains the intersection of a countable number of open dense sets, e.g., the irrational numbers form a residual subset of the real numbers. Clearly, the intersection of a countable number of residual sets is residual. A property that holds for a residual subset of a space is called a generic property.

Let $A$ be a non-empty subset of $\{1, \ldots, n\}$ and $\mathbf{R}^{A}=\left\{\left(x_{1}, \ldots, x_{n}\right)\right.$ : $x_{i}=0$ for $\left.i \notin A\right\}$. Let $|A|=$ cardinality of $A=$ dimension of $\mathbf{R}^{A}$; and similarly for $|B|,|C|$ and $|D|$. Let $\mathbf{R}_{+}^{A}=\mathbf{R}^{A} \cap \mathbf{R}_{+}^{n}$. We state without proof the following simple lemma.

Lemma 2.1. $x \in \mathbf{R}_{+}^{n}$ is a solution of the complementarity problem defined by $f$ if and only if there are non-empty subsets $A$ and $B$ of $\{1, \ldots, n\}$ such that $A \cap B=\emptyset$ and $x \in \mathbf{R}_{+}^{A} \cap f^{-1}\left(\mathbf{R}_{+}^{B}\right)$.

We will use techniques of transversality theory, as in [1], to prove that for a residual subset of $C^{1}\left(\mathbf{R}^{n}, \mathbf{R}^{n}\right), f\left(\mathbf{R}^{A}\right)$ intersects $\mathbf{R}^{B}$ transversally where $A$ and $B$ are as in Lemma 2.1 . We will then use this fact to establish that the solution set of (1) is generically discrete.

We now introduce some notation and state some definitions to be used in this section. Let $A, B, C, D \subset\{1, \ldots, n\}, A \cup B=C \cup D=$ $\{1,2, \ldots, n\}$ and $A \cap B=C \cap D=\emptyset$. So $\mathbf{R}^{n}$ is the direct sum $\mathbf{R}^{A} \oplus \mathbf{R}^{B}$ and also the direct sum $\mathbf{R}^{C} \oplus \mathbf{R}^{D}$; i.e., each $x \in \mathbf{R}^{n}$ is uniquely expressed as $x=x_{A}+x_{B}$, where $x_{A} \in \mathbf{R}^{A}$ and $x_{B} \in \mathbf{R}^{B}$, or as $x=x_{C}+x_{D}$, where $x_{C} \in \mathbf{R}^{C}$ and $x_{D} \in \mathbf{R}^{D}$. For simplicity of notation, we abbreviate $x_{A}+x_{B}$ to $\left(x_{A}, x_{B}\right)$ and $x_{C}+x_{D}$ to $\left[x_{C}, x_{D}\right]$. Given $f \in \mathrm{C}^{1}\left(\mathbf{R}^{n}, \mathbf{R}^{n}\right)$, we write $f: \mathbf{R}^{A} \oplus \mathbf{R}^{B} \rightarrow \mathbf{R}^{C} \oplus \mathbf{R}^{D}$ as

$$
f\left(x_{A}, x_{B}\right)=\left[f_{C}\left(x_{A}, x_{B}\right), f_{D}\left(x_{A}, x_{B}\right)\right],
$$


where $f_{C}\left(x_{A}, x_{B}\right)$ is the component of $f\left(x_{A}, x_{B}\right)$ in $\mathbf{R}^{C}$. Let $\widetilde{f}_{C}: \mathbf{R}^{A} \rightarrow$ $\mathbf{R}^{C}$ denote the map $x_{A} \mapsto f_{C}\left(x_{A}, 0\right)$, and let $\mathrm{D} \widetilde{f}_{C}\left(x_{A}\right)$ be its derivative. Instead of treating $\mathrm{D} \widetilde{f}_{C}\left(x_{A}\right)$ as an $n \times n$ matrix of partial derivatives with $|D|$ rows of zeroes and $|B|$ columns of zeroes, it will be more convenient to delete these zero rows and columns and consider $\mathrm{D} \widetilde{f}_{C}\left(x_{A}\right)$ as a. $|C| \times|A|$ matrix.

Definition 2.2. Let $f: M \rightarrow N$ be a $\mathrm{C}^{1}$ map from manifold $M$ to manifold $N$. Let $U$ be a submanifold of $M$ and $V$ a submanifold of $N$. Then $f(U)$ is transversal to $V$ if and only if, for $x \in U$ and $y=f(x)$, either $y \notin V$ or $y \in V$, and the tangent space $T_{y} N$ of $y$ in $N$ is the sum (not necessarily direct sum) of the tangent space $T_{y} V$ of $y$ along $V$ and the image of the tangent space $T_{x} U$ of $x$ along $U$ under the derivative of $f$, i.e., $T_{f(x)} N=T_{f(x)} V+\mathrm{D} f(x)\left(T_{x} U\right)$.

Thus, given a $C^{1} \operatorname{map} f: \mathbf{R}^{A} \oplus \mathbf{R}^{B} \rightarrow \mathbf{R}^{C} \oplus \mathbf{R}^{D}$ and $U \subset \mathbf{R}^{A}, f(U)$ is transversal to $\mathbf{R}^{D}$ if, whenever $x_{A} \in U$ and $f_{C}\left(x_{A}, 0_{B}\right)=0$, the rank of $\mathrm{D} \widetilde{f}_{C}\left(x_{A}\right)$ is $|C|$.

Definition 2.3. Given a $\mathrm{C}^{1}$ map $f: M \rightarrow N$ as above with $y \in N, y$ is a critical value of $f$ if $y$ is in the range of $f$ and for some $x$ with $f(x)=y$, $\mathrm{D} f(x)$ has rank less than the dimension of $N$. All $y$ in $N$ that are not critical values are called regular values of $f$.

Sard's Density Theorem [1, p. 37; 19, p. 72] asserts that under suitable differentiability conditions on $f$ (i.e., $f$ is $r$ times continuously differentiable, where $r>\max \{0, \operatorname{dim} M-\operatorname{dim} N\}$ ), the set of all critical values of $f$ has measure zero in $N$. Lemma 2.4 below is similar to the Transversal Density Theorem [1, p. 48], a strong consequence of Sard's Theorem. Since we have a simpler hypothesis and a different differentiability requirement than does the usual Transversal Density Theorem, we shall use Sard's Theorem to prove the exact lemma we need. In addition, by our proof of Lemma 2.4, Theorem 3.5 will be valid if we consider only maps $f$ that are affine.

Lemma 2.4. Let $A, B, C, D$ be subsets of $\{1,2, \ldots, n\}$. with $A \cap D$ $=\emptyset$ and $\mathbf{R}^{A} \oplus \mathbf{R}^{B}=\mathbf{R}^{n}=\mathbf{R}^{C} \oplus \mathbf{R}^{D}$. Then

$$
F \equiv\left\{f \in \mathrm{C}^{1}\left(\mathbf{R}^{n}, \mathbf{R}^{n}\right): f\left(\mathbf{R}^{A}\right) \text { is transversal to } \mathbf{R}^{D}\right\}
$$


is a residual subset of $\mathrm{C}^{1}\left(\mathbf{R}^{n}, \mathbf{R}^{n}\right)$. If $A \cup D \neq\{1,2, \ldots, n\}$, then $f \in F$ implies $f\left(\mathbf{R}^{A}\right) \cap \mathbf{R}^{D}=\emptyset$.

Proof. Write $\mathbf{R}^{n}=\mathbf{U}_{i=1}^{\infty} U_{i}$, where each $U_{i}$ is compact and contained in the interior of $U_{i+1}$. We will show that

$$
F_{i} \equiv\left\{f: f\left(U_{i} \cap \mathbf{R}^{A}\right) \text { is transversal to } \mathbf{R}^{D}\right\}
$$

is open and dense in $\mathrm{C}^{1}\left(\mathbf{R}^{n}, \mathbf{R}^{n}\right)$. Since $\bigcap_{i=1}^{\infty} F_{i}=F$, this will prove Lemma 2.4 .

(i) Suppose $A \cup D=\{1,2, \ldots, n\}$. Then, $\mathbf{R}^{A} \equiv \mathbf{R}^{C}$ and $\mathbf{R}^{B} \equiv \mathbf{R}^{D}$.

(a) To see that $F_{i}$ is open, let $f \in F_{i}$. For each $x_{A} \in U_{i} \cap \mathbf{R}^{A}$ with $\tilde{f}_{C}\left(x_{A}\right)=0$ in $\mathbf{R}^{C}$, the $|C| \times|C|$ matrix $\mathrm{D} \tilde{f}_{C}\left(x_{A}\right)$ has non-zero determinant. Since $f\left(U_{i} \cap \mathbf{R}^{A}\right) \cap \mathbf{R}^{D}$ is compact, these determinants are bounded away from zero. If $g$ is $\mathrm{C}^{1}$ near $f$, then not only will $g$ be pointwise near $f$, but also det $\tilde{\mathrm{D}}_{C}\left(x_{A}\right)$ will be near $\operatorname{det} \widetilde{\mathrm{D}}_{C}\left(x_{A}\right)$ for all $x_{A} \in U_{i} \cap \mathbf{R}^{A}$. So, if $g$ is $C^{1}$ near enough to $f$, then $\operatorname{det} \widetilde{D}_{C}\left(x_{A}\right)$ will be non-zero for $x_{A} \in U_{i} \cap \mathbf{R}^{A} \cap g^{-1}\left(\mathbf{R}^{D}\right)$ and $g$ will be in $F_{i}$. So $F_{i}$ is open.

(b) To see that $F_{i}$ is dense, we suppose $f \notin F_{i}$ and show that an arbitrary small perturbation of $f$ is in $F_{i}$. For some $x_{A} \in \mathbf{R}^{A}$, the rank of $\mathrm{D} \tilde{f}_{C}\left(x_{A}\right)$ is less than $|C|$ and $\tilde{f}_{C}\left(x_{A}\right)=0$ in $\mathbf{R}^{C}$, i.e., 0 is a critical value of $\widetilde{f}_{C}: \mathbf{R}^{A} \rightarrow \mathbf{R}^{C}$. By Sard's Density Theorem, there is a $v$ in $\mathbf{R}^{C}$ as close as one wishes to 0 with $v$ a regular value of $\widetilde{f}_{C}$, i.e., $\mathrm{D} \widetilde{f}_{C}\left(y_{A}\right)$ has rank $|C|$ for all $y_{A}$ in $\tilde{f}_{C}^{-1}(v)$. Define $g \in C^{1}\left(\mathbf{R}^{n}, \mathbf{R}^{n}\right)$ by

$$
g(x)=\left[f_{C}(x)-v, f_{D}(x)\right] \text { for all } x \in \mathbf{R}^{n} .
$$

If $x_{A} \in \mathbf{R}^{A}$ is such that $g_{C}\left(x_{A}, 0\right)=0$ in $\mathbf{R}^{C}$, then $f_{C}\left(x_{A}, 0\right)=v$ and $\tilde{D}_{C}\left(x_{A}\right)=\mathrm{D} \tilde{f}_{C}\left(x_{A}\right)$. If $v$ is a regular value of $\tilde{f}_{C}$, then 0 is a regular value of $\tilde{g}_{C}$. So $g \in F_{i}$. By taking $v$ as close to 0 as one chooses, one can make $g$ as $C^{1}$-close to $f$ as one wishes.

(ii) Suppose $A \cup D \neq\{1,2, \ldots, n\}$. Then $|A|+|D|<n$ and $|A|<|C|$. So the $|C| \times|A|$ matrix $\widetilde{D}_{C}\left(x_{A}\right)$ has rank less than $|C|$ for all $x_{A}$ in $\mathbf{R}^{A}$ and so each element of $f_{C}\left(\mathbf{R}^{A}\right)$ is critical.

(a) To see that $F_{i}$ is open, let $f \in F_{i}$. Since the rank of $\mathrm{D} \tilde{f}_{C}(x)$ is less than $\mid C l, f\left(U_{i} \cap \mathbf{R}^{A}\right) \cap \mathbf{R}^{D}=\emptyset$. Since $f\left(U_{i} \cap \mathbf{R}^{D}\right)$ is compact, for all $g$ $\mathrm{C}^{0}$-near $f$, we have $g\left(U_{i} \cap \mathbf{R}^{A}\right) \cap \mathbf{R}^{D}=\emptyset$ and $g \in F_{i}$. So $F_{i}$ is open.

(b) To see that $F_{i}$ is dense, follow argument (i)(b) now with $v$ not in the image of $\tilde{f}_{C}$. Then 0 will not be in the image of $\tilde{g}_{C}$. (Such a $v$ exists again because Sard's Theorem asserts that $f_{C}\left(\mathbf{R}^{A}\right)$ has measure zero in $\mathbf{R}^{C}$ for $|A|<|C|$.) 
The final assertion of the lemma follows from arguments in (ii)(a).

Theorem 2.5. For some residual set of $g$ in $\mathrm{C}^{1}\left(\mathbf{R}^{n}, \mathbf{R}^{n}\right)$, the solution set for the corresponding complementarity problem is discrete.

Proof. Let $A, D$ be subsets of $\{1,2, \ldots, n\}$ with $A \cap D=\emptyset$. Let

$$
F^{A, D}=\left\{f \in \mathrm{C}^{1}\left(\mathbf{R}^{n}, \mathbf{R}^{n}\right): f\left(\mathbf{R}^{A}\right) \text { is transversal to } \mathbf{R}^{D}\right\} .
$$

Let $G$ be the intersection of the $F^{A, D}$ for all such pairs $A, D$. Since each $F^{A, D}$ is a residual set, $G$ is a residual subset of $C^{1}\left(\mathbf{R}^{n}, \mathbf{R}^{n}\right)$. If $f \in F^{A, D}$ and $A \cup D \neq\{1,2, \ldots, n\}$, then $\mathbf{R}^{A} \cap f^{-1}\left(\mathbf{R}^{D}\right)$ is empty: If $A \cup D$ $=\{1,2, \ldots, n\}$ and $x \in \mathbf{R}^{A} \cap f^{-1}\left(\mathbf{R}^{D}\right)$, then $A \equiv C, \widetilde{f}_{C}: \mathbf{R}^{A} \rightarrow \mathbf{R}^{A}, \tilde{f}_{C}(x)$ $=0$, and $\mathrm{D} \tilde{f}_{C}(x)$ is non-singular. By the Inverse Function Theorem [18, p. 35], there is an open set $W \subset \mathbf{R}^{A}$ around $x$ on which $\widetilde{f}_{C}$ is a diffeomorphism (one-to-one and onto $f(W)$ ); and so $x$ is the only point in $W$ with $\tilde{f}_{C}(x)=0$, i.e., $x$ is the only point in $\mathbf{R}^{A} \cap f^{-1}\left(\mathbf{R}^{D}\right)$. Therefore $\mathbf{R}^{A} \cap f^{-1}\left(\mathbf{R}^{D}\right)$ is discrete. (It clearly may be an infinite set.) By Lemma 2.1, such $\mathbf{R}^{A} \cap f^{-1}\left(\mathbf{R}^{D}\right)$ are the solution sets for $f$. (Alternatively, see $[1$, p. 45].)

Since $x+f(x)>0$ for a solution $x$ is equivalent to $A \cup D=\{1,2, \ldots, n\}$ and $A \cap D=\emptyset$ for each pair $A$ and $D$ for which $x \in \mathbf{R}^{A} \cap f^{-1}\left(\mathbf{R}^{D}\right)$, and since $\mathrm{D} \tilde{f}_{C}(x)$ is the principal minor of $\mathrm{D} f(x)$ corresponding to the indices for which $x_{i}>0$, Theorem 2.5 can be restated as follows:

Corollary 2.6. Let $f \in \mathrm{C}^{1}\left(\mathbf{R}_{+}^{n}, \mathbf{R}^{n}\right)$. Suppose each solution $x \in \mathbf{R}_{+}^{n}$ to (1) has the following properties:

(i) $x+f(x)>0$;

(ii) if $B \subset\{1, \ldots, n\}$ is the set of indices $i$ such that $x_{i}>0$, the principal minor of the matrix $\mathrm{D} f(x)$ corresponding to $B$ is non-zero. Then the solution set of (1) is discrete and the set of all such maps $f$ is a residual subset of $\mathrm{C}^{1}\left(\mathbf{R}_{+}^{n}, \mathbf{R}^{n}\right)$.

Remark 2.7. The same proof works if instead of $C^{1}\left(\mathbf{R}^{n}, \mathbf{R}^{n}\right)$ we consider affine maps and gives us an alternative proof for the linear complementarity problem. However, if we were considering only continuous maps $\mathbf{R}_{+}^{n} \rightarrow \mathbf{R}^{n}$, we would have to weaken "residual" to "dense" in the statement of Theorem 2.5. For, in the proof of Lemma 2.4, although parts (i)(b), (ii)(a), (ii)(b) carry over automatically in $\mathrm{C}^{0}\left(\mathbf{R}^{n}, \mathbf{R}^{n}\right)$, the 
openness proof in (i)(a) does not. In fact, if $x$ is a solution of the complementarity problem defined by $f$, there is a $g$ arbitrarily $\mathrm{C}^{0}$-close to $f$ with the property that an interval of points around $x$ is part of the solution set for $g$.

\section{3}

In this section, we shall prove that if $f$ satisfies the generic non-degeneracy conditions of Corollary 2.6 near solutions of (1) and also a condition sufficient for the existence of a solution [13], then (1) has an odd number of solutions. This generalizes a result of Lemke [14] for the linear problem. Our principal tool, however, will be the fixed point index $[7,5]$. To do this, we must first state (1) as a fixed point problem.

Define $h_{f}: \mathbf{R}_{+}^{n} \rightarrow \mathbf{R}_{+}^{n}$ by

$$
h_{f}(x)=\arg \min \left\{\|v-x+f(x)\|^{2}: v \geq 0\right\} \text {, }
$$

i.e., the non-negative vector $v$ which minimizes $\|v-x+f(x)\|^{2}$. Then $h=h_{f}$ is well-defined since by the usual calculus techniques one can show that $h=\left(h_{1}, \ldots, h_{n}\right)$, where

$$
h_{i}(x)= \begin{cases}0 & \text { if } f_{i}(x)-x_{i} \geq 0 \\ x_{i}-f_{i}(x) & \text { if } f_{i}(x)-x_{i} \leq 0\end{cases}
$$

Lemma 3.1. The following statements are equivalent:

(i) $x$ satisfies (1);

(ii) $x \in \Gamma(x) \equiv \arg \min \{\langle v, f(x)\rangle: v \geq 0\}$;

(iii) $h_{f}(x)=x$.

Proof. See [10].

We now state a condition which Karamardian [13] has shown to be sufficient for the existence of a solution to (1).

(K) There is a compact set $C \subset \mathbf{R}_{+}^{n}$ such that for each $x \in \mathbf{R}_{+}^{n} \backslash C$ there is a $y \in C$ with $\langle y-x, f(x)\rangle<0$.

Lemma 3.2. If $f$ satisfies condition $(\mathrm{K})$, then all the fixed points of $h_{f}$ lie in the compact set $C$ of $(\mathrm{K})$. 
Proof. Let $x \in \mathbf{R}_{+}^{n} \backslash C$. By condition $(\mathrm{K}),\langle y, f(x)\rangle\langle\langle x, f(x)\rangle$ for some $y \in C$. Therefore, $x \notin \Gamma(x)$, where $\Gamma$ is as in Lemma 3.1. So $h_{f}(x) \neq x$ by Lemma 3.1 .

Lemma 3.3. Suppose $f$ satisfies condition (K) for some compact set $C$ and let $h=h_{f}$. Then there is a compact convex set $D$ and a map $g: \mathbf{R}_{+}^{n} \rightarrow D$ such that

(i) $C \subset D$;

(ii) $g|C=h| C$;

(iii) fixed point set of $g=$ fixed point set of $h$.

Proof. Let $D \subset \mathbf{R}_{+}^{n}$ be any compact convex set containing $C \cup h(C)$. Define $g: \mathbf{R}_{+}^{n} \rightarrow D$ by

$$
g(x)=\arg \min \left\{\|y-x+f(x)\|^{2}: y \in D\right\},
$$

i.e., $g(x)$ is that $y$ in $D$ that minimizes $\|y-x+f(x)\|^{2}$. Then $g$ is welldefined since $D$ is compact and convex.

Let $x \in C$. Since $h(x) \in D$, we have $g(x)=h(x)$, and (ii) follows.

To prove (iii), it is sufficient to show that all the fixed points of $g$ lie in $C$. Since $g\left(\mathbf{R}_{+}^{n}\right) \subset D$, all fixed points of $g$ lie in $D$. Let $x \in D \backslash C$. By the Kuhn-Tucker Theorem [20], if $x$ minimizes $\|y-x-f(x)\|^{2}$ over $D$, then $\langle v-x, f(x)\rangle \geq 0$ for all $v$ in $D$. But by $(\mathrm{K})$ there is a $y \in C \subset D$ such that $\langle y-x, f(x)\rangle<0$. Hence no $x$ in $D \backslash C$ can be a fixed point of $g$, and (iii) follows.

The primary tool in the proof of Theorem 3.5 below is the fixed point index of a mapping. Let $H_{j}(M)$ denote the $j^{\text {th }}$ (singular) homology group of manifold $M$ over the integers $\mathrm{Z}$. For $N \subset M$, let $H_{j}(M, N)$ be the corresponding relative homology group. For example, if $S^{m}$ is the $m$-dimensional sphere, $H_{j}\left(\mathrm{~S}^{m}\right)=\mathbf{Z}$ for $j=0$ or $m$ and $H_{j}\left(\mathrm{~S}^{m}\right)=\{0\}$ for all other $j$. The element in $H_{m}\left(\mathrm{~S}^{m}\right)$ corresponding to $1 \in \mathbf{Z}$ is called the fundamental class $\gamma\left(\mathrm{S}^{m}\right)$. A continuous map $G: M_{1} \rightarrow M_{2}$ induces group homomorphisms $H_{j}(G): H_{j}\left(M_{1}\right) \rightarrow H_{j}\left(M_{2}\right)$ for all $j$. If $G$ is a homeomorphism, each $H_{j}(G)$ is an isomorphism. If $G: \mathrm{S}^{m} \rightarrow \mathrm{S}^{m}, H_{m}(G)$ is a homomorphism of the integers. So $H_{m}(G)\left(\gamma\left(\mathrm{S}^{m}\right)\right)=d \gamma\left(\mathrm{S}^{m}\right)$ for some integer $d$, called the degree of $G$. Roughly speaking, the degree of $G$ measures how many times the image of $G$ wraps around $\mathrm{S}^{m}$.

Let $\mathbf{Z}$ be an isolated fixed point of $G: \mathbf{R}^{n} \rightarrow \mathbf{R}^{n}$. Choose the disk $D \equiv\left\{x \in \mathbf{R}^{n}:|x-z| \leq r\right\}$ of radius $r$ about $z$ containing no other fixed 
points of $G$. Let $S^{n-1}$ be the boundary of $D$. The map

$$
\bar{G}(x)=r(G(x)-x) /|G(x)-x|+z \text { for } x \in \mathrm{S}^{n-1}
$$

takes $S^{n-1}$ to $S^{n-1}$. Its degree is called the index of the fixed point $z$, $I_{G}(z)$. If $G$ has a finite number of fixed points $\left\{z^{1}, \ldots, z^{k}\right\}$, the fixed point index of $G, I_{G}$, is the sum of the indices of the fixed points $I_{G}=\sum_{i=1}^{k} I_{G}\left(z^{i}\right)$. More generally, if $U$ is an open subset of $\mathbf{R}^{n}$ and $G: U \rightarrow \mathbf{R}^{n}$ has a compact fixed point set $F_{G}$, then $H_{n}\left(U, U \backslash F_{G}\right)$ and $H_{n}\left(\mathbf{R}^{n}, \mathbf{R}^{n} \backslash\{0\}\right)$ are both isomorphic to the integers $\mathbf{Z}$ and thus each has a "fundamental class" $\gamma$. Since

$$
\text { id-G: }\left(U, U \backslash F_{G}\right) \rightarrow\left(\mathbf{R}^{n}, \mathbf{R}^{n} \backslash\{0\}\right)
$$

$H_{n}(\mathrm{id}-G)$ is a homomorphism of the integers and

$$
H_{n}(\mathrm{id}-G) \gamma\left(U, U \backslash F_{G}\right)=I_{G} \gamma\left(\mathbf{R}^{n}, \mathbf{R}^{n} \backslash\{0\}\right)
$$

for some integer $I_{G}$ (the fixed point index of $G$ ). As shown in [7] and [17], the fixed point index has the following properties for $G: U \rightarrow \mathbf{R}^{n}$ :

(I.1) If $F_{G}$ is a finite set, both definitions above yield the same integer. In particular, if $U=\bigcup_{j=1}^{m} U_{j}$, where the $U_{j}$ are open, then $I_{G}=$ $\sum_{j=1}^{m} I_{G \mid U_{\dot{j}}}$

(I.2) If $G$ maps all of $U$ to the point $p \in \mathbf{R}^{n}$, then $I_{G}=+1$ if $p \in U$ and $I_{G}=0$ if $p \notin U$.

(I.3) If $G_{t}: U \rightarrow \mathbf{R}^{n}$ for $0 \leq t \leq 1$ is a continuous family of maps and if $\mathrm{U}_{t} F_{G_{t}}$ is compact, then $I_{G_{0}}=I_{G_{1}}$.

(I.4) If $F_{G} \subset K \subset W \subset U$, where $K$ is compact and $W$ open, then

$$
H_{n}(\mathrm{id}-G) \gamma(W, W \backslash K)=I_{G} \gamma\left(\mathbf{R}^{n}, \mathbf{R}^{n} \backslash\{0\}\right) .
$$

(I.5) If $G: V \rightarrow \mathbf{R}_{+}^{n}$, where $V=W \cap \mathbf{R}_{+}^{n}$ for some open $W$ in $\mathbf{R}^{n}$, let $i: \mathbf{R}^{n} \rightarrow \mathbf{R}^{n}$ be the inclusion and $r: \mathbf{R}^{n} \rightarrow \mathbf{R}_{+}^{n}$ be a retract, i.e., a map with $r \circ i=$ identity on $\mathbf{R}_{+}^{n}$. Then $I_{G}$ is defined as $I_{i}{ }^{\circ} G^{\circ} r ; I_{G}$ is independent of choice of $r$ and satisfies (I.1)-(I.4).

Lemma 3.4. Assume that $f \in C^{1}\left(\mathbf{R}_{+}^{n}, \mathbf{R}^{n}\right)$ satisfies the generic conditions (i) and (ii) of Corollary 2.6. Let $h_{f}$ be as defined in (2). Every fixed point of $h_{f}$ has index \pm 1 . 
Proof. Extend $f$ to a $\mathbf{C}^{1}$ function on all of $\mathbf{R}^{n}$ as in Section 2. Then (3) defines an extension $\widetilde{h}$ of $h_{f}$ to all of $\mathbf{R}^{n}$, whose range is still $\mathbf{R}_{+}^{n}$. Let $y$ be a fixed point of $h_{f}$. By Corollary 2.6 and Lemma 3.1, $f(y)+y>0$. So $f_{j}(y)=0$ if and only if $y_{j}>0$ for each component $j=1, \ldots, n$. By (3), $\widetilde{h}$ is $\mathrm{C}^{1}$ in some neighborhood $W$ of $y$. Now $y \in \mathbb{R}^{A} \cap \tilde{f}^{-1}\left(\mathbf{R}^{B}\right)$, where $\mathbf{R}^{n}=\mathbf{R}^{A} \oplus \mathbf{R}^{B}$. So $y=\left(y_{A}, 0_{B}\right), f=\left(f_{A}, f_{B}\right)$, and $\widetilde{h}=\left(\widetilde{h}_{A}, \widetilde{h}_{B}\right)$. On $W$,

$$
\tilde{h}_{A}\left(x_{A}, x_{B}\right)=x_{A}-f_{A}\left(x_{A}, x_{B}\right) \in \mathbf{R}^{A}
$$

and $\widetilde{h}_{B} \equiv 0$, i.e.,

$$
\operatorname{id}-\tilde{h}\left(x_{A}, x_{B}\right)=\left(f_{A}\left(x_{A}, x_{B}\right), x_{B}\right) .
$$

Therefore,

$$
\mathrm{D}(\mathrm{id}-\tilde{h})(y)=\left(\begin{array}{ll}
\mathrm{D}_{A}\left(y_{A}\right) & 0 \\
0 & I
\end{array}\right)
$$

By Corollary 2.6, $\mathrm{D} \tilde{f}_{A}\left(y_{A}\right)$ is non-singular. So, $\mathrm{D}(\mathrm{id}-\widetilde{h})(y)$ is non-singular. By the Inverse Function Theorem again, there is an open set $V \subset W$ containing $y$ with (id- $-\tilde{h}) \mid V$ a smooth homeomorphism onto its image. Choose $V$ to be a ball of radius $\epsilon$ about $y, 0<\epsilon<1$. Therefore $H_{n}(\mathrm{id}-\widetilde{h}): H_{n}(V, V y) \rightarrow H_{n}\left(\mathbf{R}^{n}, \mathbf{R}^{n} \backslash\{0\}\right)$ is an isomorphism of the integers and sends +1 to \pm 1 . So $I_{\widetilde{h} \mid V}= \pm 1$.

However, our goal is to compute $I_{h_{f}}(y)=I_{h_{f} \mid V \cap \mathbf{R}_{+}^{n}}$. Let $p^{t}: \mathbf{R}^{n} \rightarrow \mathbf{R}^{n}$ be the following homotopy, for $0 \leq t \leq 1$ :

$$
p^{t}=\left(p_{1}^{t}, \ldots, p_{n}^{t}\right), \quad p_{j}^{t}\left(x_{1}, \ldots, x_{n}\right)= \begin{cases}x_{j} & \text { if } x_{j} \geq-t \\ -t & \text { if } x_{j} \leq-t\end{cases}
$$

$p^{0}$ is a retract of $\mathbf{R}_{+}^{n}$. Since $V$ is a ball of radius $\epsilon$ about $y, \widetilde{h} \circ p^{\epsilon}|V=\widetilde{h}| V$. Since $\widetilde{h}\left(\mathbf{R}^{n}\right) \subset \mathbf{R}_{+}^{n}$ and (id- $\left.\widetilde{h}\right) \mid V$ is a homeomorphism, $y$ is the only fixed point of $\widetilde{h}{ }^{\circ} p^{t}$ in $V$ for $0 \leq t \leq \epsilon$. Thus

$$
\begin{array}{rlrl}
I_{h \mid V \cap \mathrm{R}_{+}^{n}} & \equiv I_{i^{\circ} h^{\circ} p^{0} \mid V} & & (\text { by }(\mathrm{I} .5)) \\
& =I_{i^{\circ} \widetilde{h}^{\circ} p^{0} \mid V} & & (\text { since } h \circ p=\widetilde{h} \circ p) \\
& =I_{i^{\circ} \widetilde{h}^{\circ} p^{e} \mid V} & & (\text { by }(\mathrm{I} .3)) \\
& =I_{\widetilde{h} \mid V}= \pm 1 . &
\end{array}
$$


Theorem 3.5. Let $f \in \mathrm{C}^{1}\left(\mathbf{R}_{+}^{n}, \mathbf{R}^{n}\right)$ and let (1) denote the complementarity problem corresponding to $f$. Suppose $f$ satisfies the generic conditions (i) and (ii) of Corollary 2.6 and also condition (K) for the existence of a solution to (1). Then (1) has an odd number of solutions.

Proof. Let $h=h_{f}$ as in Lemma 3.1. Let $C$ be the compact set of condition $(\mathrm{K})$. Without loss of generality, one can take $C$ to be the closure of an open set in $\mathbf{R}_{+}^{n}$. Let $D \supset C$ and $g: \mathbf{R}_{+}^{n} \rightarrow D$ be as described in Lemma 3.3. Fix $\bar{x} \in C$ and define the homotopy $H_{t}: \mathbf{R}_{+}^{n} \rightarrow \mathbf{R}^{n}$ by

$$
H_{t}(x)=(1-t) g(x)+t \bar{x}
$$

for $t \in[0,1] ; H_{0}=g$ and $H_{1} \equiv \bar{x}$. Since $D$ is convex, $H_{t}\left(\mathbf{R}_{+}^{n}\right) \subset D$. Let $K=\mathrm{U}_{t}\left\{x: H_{t}(x)=x\right\}$. Then $K$ is compact since $g\left(\mathbf{R}^{n}\right) \subset D$ and $D$ is compact. By Lemma 3.3, the fixed point set $F_{h}$ of $h$ equals $F_{g}$ and is compact. Since $h=g$ in a neighborhood of $F_{h}$ in $\mathbf{R}_{+}^{n}, I_{h}=I_{g}$ by (I.4). By (I.2) and (I.3), $I_{g}=I_{H_{1}}=+1$. By Theorem 2.5 and Lemma 3.2, $F_{h}$ is discrete and compact. Thus $F_{h}$ is a finite set, say $\left\{y^{1}, \ldots, y^{m}\right\}$. By Lemma $3.4, I_{h}\left(y^{i}\right)= \pm 1$ for each $i$. By (I.1), $I_{h}=\sum_{i=1}^{m} I_{h}\left(y^{i}\right)$. Therefore, since 1 is the sum of $m$ real numbers of absolute value $1, m$ is odd.

An interesting class of maps that satisfy condition (K) : tre the strongly co-positive maps, i.e., $f: \mathbf{R}_{+}^{n} \rightarrow \mathbf{R}^{n}$ with $\langle f(x)-f(0), x\rangle \geq \alpha\langle x, x\rangle$ for some $\alpha>0$ and all $x$. In this case, the compact set $C$ needed for condition $(\mathrm{K})$ is $\left\{x: x \leq \alpha^{-1}\|f(0)\|\right\}$, as shown in [13].

Corollary 3.6. If $f: \mathbf{R}_{+}^{n} \rightarrow \mathbf{R}^{n}$ is strongly co-positive and non-degenerate (in the sense of Theorem 2.5), then (1) has an odd number of solutions.

An example of such a map is any square matrix with all entries positive and all principal minors non-zero. This corollary can be considered as a generalization of a result of Lemke [14] for co-positive matrices in the linear complementarity problem.

\section{References}

[1] R. Abraham and J. Robbin, Transversal mappings and flows (Benjamin, New York, 1967).

[2] R.W. Cottle, "Nonlinear programs with positively bounded jacobians", SIAM Journal on Applied Mathematics 14 (1963) 147-158. 
[3] R.W. Cottle and G.B. Dantzig, "Complementary pivot theory of mathematical programming", Linear Algebra and Its Applications 1 (1968) 103-125.

[4] G. Debreu, "Economies with a finite set of equilibrium", Econometrica 38 (1970) 387392.

[5] E. Dierker, "Two remarks on the number of equilibria of an economy", Working Paper CP-333, Center for Research in Management Science, University of California, Berkeley, (May 1971).

[6] E. Dierker and H. Dierker, "On the local uniqueness of equilibria", Econometrica, to appear.

[7] A. Dold, "Fixed point index and fixed point theorem for euclidean neighborhood retracts", Topology 4 (1965) 681-689.

[8] P. Du Val, "The unloading problem for plane curves", American Journal of Mathematics 62 (1940) 307-317.

[9] B.C. Eaves, "The linear complementarity problem in mathematical programming", Management Science 17 (1971) 612-634.

[10] B.C. Eaves, "On the basic theorem of complementarity", Mathematical Programming 1 (1971) $68-75$.

[11] A.W. Ingleton, "A problem in linear inequalities", Proceedings of the London Mathematical Society $3^{\text {rd }}$ Series, 16 (1966) 519-536.

[12] S. Karamardian, "The non-linear complementarity problem with applications, Parts 1 and 2," Journal of Optimization Theory and Applications 4 (1969) 87-98, 167-181.

[13] S. Karamardian, "The complementarity problem", Mathematical Programming 1 (1972) $107-129$.

[14] C.E. Lemke, "Bimatrix equilibrium points and mathematical programming", Management Science 11 (1965) 681-689.

[15] K.G. Murty, "On the number of solutions to the complementary problemand the spanning properties of complementary cones", Linear Algebra and its Applications 5 (1972) 65108.

[16] R. Saigal, "A characterization of the parity of the number of solutions to the linear complementarity problem", SIAM Journal on Applied Mathematics 23 (1972) 40-45.

[17] J. Schwartz, Non-linear functional analysis (Gordon and Breach, New York, 196?).

[18] S. Smale, "Global analysis in economics, I, pareto optimum and a generalization of Morse theory", in: Proceedings of the 1971 Dynamical Systems Symposium, Salvador, Brasil, to appear.

[19] M. Spivak, Calculus on manifolds (Benjamin, New York, 1965).

[20] W.I. Zangwill, Non-linear programming (Prentice Hall, Englewood Cliffs, N.J., 1967). 